\title{
Optical-fiber ring cavity with saturable rare-earth-doped fiber
}

\author{
E. Aguilar, S. Stepanov*, and E. Hernández \\ Optics, CICESE, Ensenada, BC, 22860, México. \\ *e-mail: steps@cicese.mx
}

Received 24 October 2020; accepted 21 November 2020

\begin{abstract}
Resonance properties of the all-fiber ring cavity filled with $\chi^{\prime \prime \prime}$ nonlinear material - saturable rare-earth-doped fiber are analyzed and experimentally investigated. Unlike the earlier investigated erbium-doped fiber at $1550 \mathrm{~nm}$ where the optical absorption photo-induced change (saturation) is observed only, the ytterbium-doped fiber at $1064 \mathrm{~nm}$ demonstrates the saturation of the refractive index mainly. For this configuration we report the experimental observation of the optical bistability and hysteresis in the transmitted output light at the $10 \mathrm{~mW}$ scale incident light power. The experimental results are in qualitative agreement with the theoretical analysis that takes into account the saturation of both parameters: the optical absorption and the refractive index of the doped fiber. The reported results seem very promising for applications in high-sensitivity interferometric configurations at $1064 \mathrm{~nm}$ operation wavelength.
\end{abstract}

Keywords: Rare-earth doped fibers; optical bistability; optical interferometry.

PACS: 42.60Da; 42.65.Wi; 42.65.Pc

\section{Introduction}

High-quality optical-fiber ring cavities can be arranged in a very simple way by the connection of two opposite terminals of a single-mode optical fiber coupler with division ratio $\kappa$-see Fig. 1. The resonance properties of this configuration are similar to those of a bulk Fabry Perot (FP) cavity with the back $100 \%$-reflection mirror and were analyzed earlier see $e . g$. $[1,2]$. This cavity demonstrates the conventional FP set of the equally spaced by $\Delta f=c / n_{\mathrm{eff}} L$ (where $L$ is the closed-loop length and $n_{\text {eff }}$ is the effective refractive index of the fiber mode) resonance transmittance minima.

Different versions of the ring fiber cavity are widely utilized in the single-frequency fiber lasers [3]. As itself, this arrangement is also interesting for different applications as a filter, optical memory, optical delay line, etc. [4,5]. Probably, one of the most interesting properties of this cavity is the existence of a critical coupling $\kappa=t$ ( $t$ is the total transmittance of the closed-loop), for which the resonance transmitted light power goes to zero: $\left|E_{2}\right|^{2}=0$. This, clearly, means that under this condition, the total incident light power is absorbed

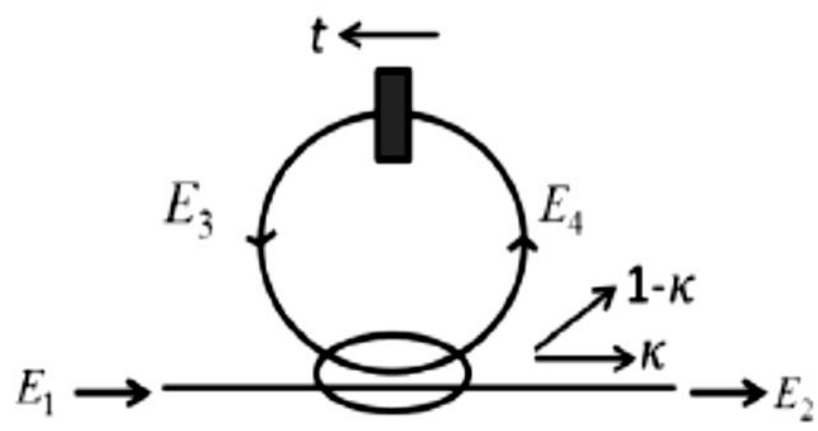

FIGURE 1. Ring optical fiber cavity ( $t$ is the total transmittance of the loop and $\kappa$ is the coupler division rate). inside the cavity. Also, the light power entering the closedloop is maximum in this case and can be significantly higher than the incident one. Figure 2 that is taken from our earlier publication [5] shows the results of a numerical simulation of the spectral profiles of the normalized output and in-cavity powers around one resonance peak, evaluated for $\kappa=0.5$ and three different values of $t=0.3,0.5$, and 0.7 .

What is also interesting is that around the point of a critical coupling point when one goes from the under-coupling $(\kappa>t)$ to the over-coupling $(\kappa<t)$, the dispersion in the resonance point changes its sign and the "slow" light propagation is substituted by the "fast" light propagation [4]. This opens interesting possibilities for manipulation with optical pulses [4]. Especially promising can be the possibility to control the pulse delay by optical means, e.g., by changing the average incident light intensity. Such results were reported in [6] using a saturable semiconductor amplifier, incorporated in the fiber ring cavity, and in [5] using the saturable erbiumdoped fiber (EDF).

The experiments reported in [5] were performed with the saturable EDF at the wavelength of $1550 \mathrm{~nm}$. At this particular wavelength, the photoinduced refractive index change of the doped fiber that accompanies the saturation of its optical absorption is minimum [7] and can, practically, be neglected. In this case, a variation of the average incident light power does not result in a detuning from the incident light wavelength but changes the coupling with the cavity only. Note that even in this case, the strict analysis obviously needs a self-consistent consideration of the cavity and the saturable medium inside it.

The situation proves to be more complicated and interesting if the saturable fiber, in addition to the absorption change, also demonstrates a change of the refractive index. This leads to the photo-induced detuning of the cavity with the incident light and can result in the observation of the optical bistability 

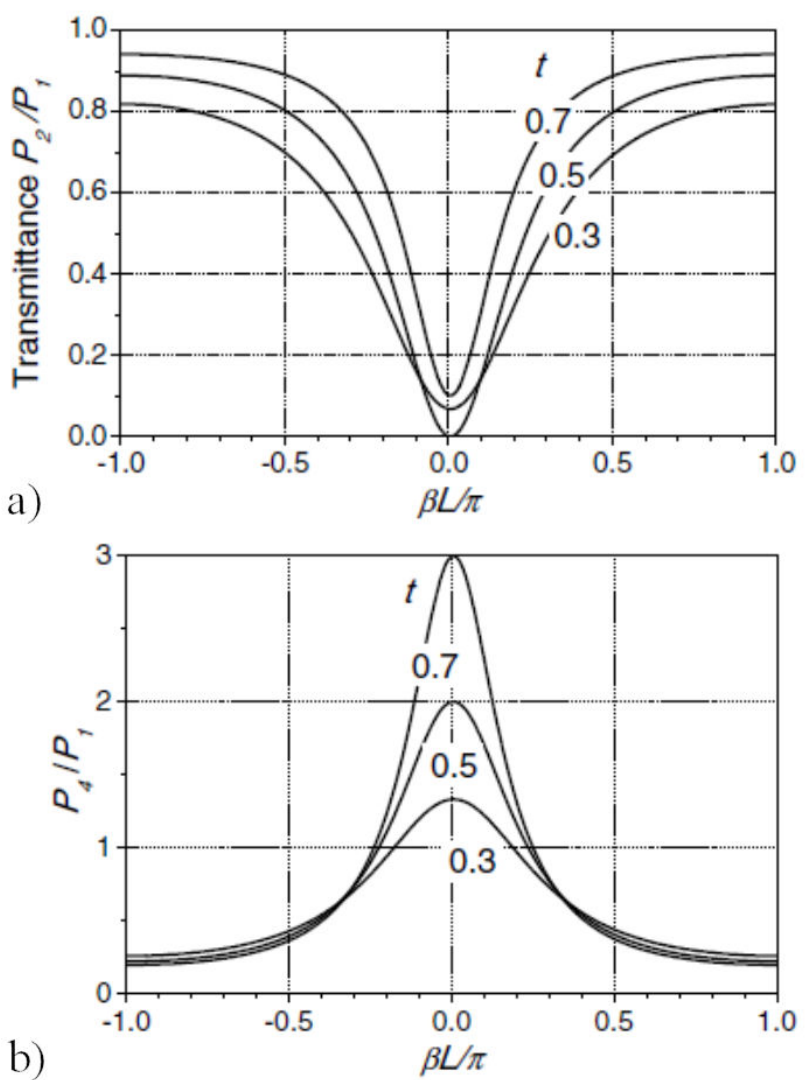

FIGURE 2. Spectral dependences of the normalized output power $P_{2} / P_{1}$-a, and the normalized light power $P_{4} / P_{1}$ that enters the closed-loop. The curves were calculated in [5] for $L=5 \mathrm{~m}$, $\lambda \approx 1550 \mathrm{~nm}$, fiber effective refractive index $n_{\text {eff }} \approx 1.5, \kappa=0.5$ for under-coupling $(t=0.3)$, critical coupling $(t=0.5)$, and overcoupling $(t=0.7)$.

$[8,9]$. The effects are manifested in the observation of two different output power values (or the transmittances $\mathrm{T}$ ) of the configuration for the same fixed incident light power. It is also demonstrated in an effect of the "optical hysteresis", i.e., in different dependences of the output power versus incident one depending on the direction of the input power change (growth or decay). A similar hysteresis loop can also be observed for a fixed incident power, but when the externally introduced detuning changes in the opposite directions.

Below, we present the results of the experimental investigation of the ring fiber-optic cavity with the saturable ytterbium-doped fiber (YDF) in it. The saturation of the optical absorption in YDF in the wavelength range $\lambda>1000 \mathrm{~nm}$ $[10,11]$ is also accompanied by a significantly larger photoinduced change of the doped fiber refractive index $\Delta n$ as compared with that of the optical absorption $\Delta \alpha(\Delta n \gg$ $\Delta \alpha \lambda / 4 \pi)$. Simplifying, we can neglect the photoinduced changes of the doped fiber absorption and consider the saturable YDF as a Kerr media with the refractive index dependent on the incident light ( $I_{4}$ in Fig. 1) intensity. The specific feature of this nonlinear medium is that it is rather inertial. The characteristic relaxation time of the refractive index to a new value corresponding to the changed light intensity occurs with the characteristic time, governed by the $Y b^{+3}$ ion metastable level relaxation time $\tau_{0}$ that is about $1 \mathrm{~ms}$ in YDF [12].

\section{Experimental configuration and results}

Resonance ring fiber cavity with the saturable YDF was investigated using the experimental setup presented in Fig. 3. In the reported experiments we utilized a $1.77 \mathrm{~m}$-long segment of the Leikki Yb-1200 ytterbium-doped single-mode fiber. Following the provider information, the fiber has the mode-filed diameter (MFD) $4.4 \pm 0.8 \mu \mathrm{m}$ and the numerical aperture (NA) 0.2 at wavelength $1060 \mathrm{~nm}$. The utilized doped fiber piece was spliced between two pieces of conventional single-mode fiber HI1060 of Corning and terminated with the standard APC connectors.

As the coherent light source, we utilized cw Nd:YAG laser CL-2000 from CrystaLaser with the maximum $\sim 300$ $\mathrm{mW}$ power at the single-mode optical fiber output. The laser wavelength is $1064 \mathrm{~nm}$ with the spectral width $\sim 10^{-5} \mathrm{~nm}$, that ensured a coherence length of about $300 \mathrm{~m}$. The utilized photodetectors were InGaAs PIN photodiodes DET410 from Thorlabs with the DC bias of $12 \mathrm{~V}$ and external load resistors $R_{L} \approx 1 \mathrm{kom}$. This ensured the constant transfer function of the input digital oscilloscope circuit up to $1 \mathrm{MHz}$.

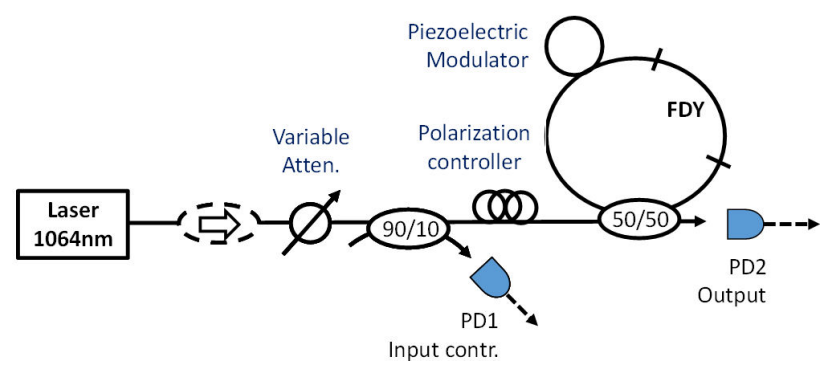

FIGURE 3. Schematic of the experimental ring optical fiber cavity with a segment of the saturable YDF.

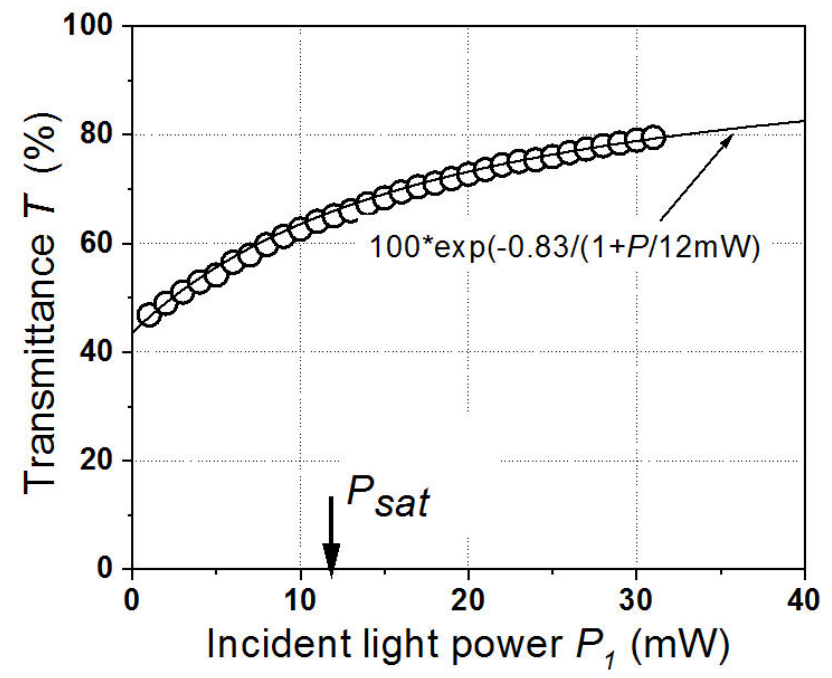

FIGURE 4. Transmittance of the utilized YDF as a function of the incident light power $(\lambda=1064 \mathrm{~nm})$. 


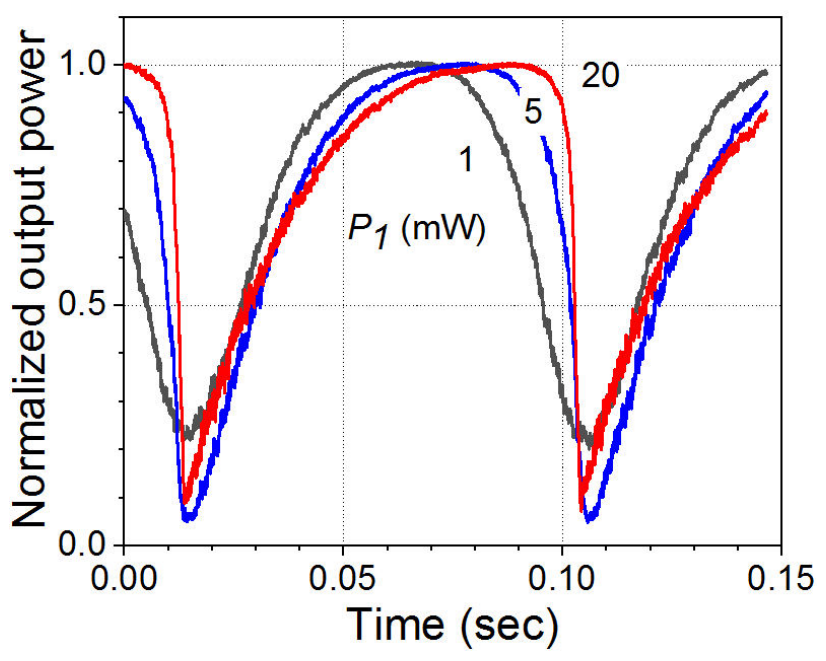

FIGURE 5. The normalized output power transmitted the resonance fiber cavity with the saturable YDF, observed with the $1.3 \mathrm{~Hz}$ triangle modulation of the cavity optical length for different incident light power $P_{1}$.

Inside the cavity loop, we have also introduced the piezoelectric phase modulator (stretcher). It consisted of the piezoelectric cylinder with several meters of the single-mode fiber HI1060 that was wound around it. Application of an external voltage to the electrodes on the opposite (external and internal) cylinder surfaces resulted in a change of its diameter that resulted in the phase modulation of the transmitted through the fiber wave.

Direct measurements performed with the doped fiber at the operation wavelength $1064 \mathrm{~nm}$ [12] resulted in the nonlinear transmittance $\mathrm{T}$ curves presented in Fig. 4. This dependence allowed us to evaluate the initial (not saturated) doped fiber absorbance $\alpha_{0} L \approx 0.83$ and the saturating power $P_{\text {sat }} \approx 12 \mathrm{~mW}$. As usually [13], the latter parameter is defined as the incident light power resulting in a two-fold reduction of initial optical absorption of the saturable medium. The above-mentioned value is in a reasonable agreement with that reported for a similar YDF at $1064 \mathrm{~nm}$ earlier [14].

Figure 5 shows the output power profiles obtained with the triangular-shape modulation of the optical length of the fiber loop using the piezoelectric phase modulator (stretcher) inside the cavity loop (Fig. 3). The modulation was performed at a rather low frequency $(<5 \mathrm{~Hz})$ to ensure quasisteady-state operation of the cavity with the saturable YDF. With the additional fiber length $(12.3 \mathrm{~m})$ inside the piezoelectric modulator, the total fiber ring length was about $\approx 16 \mathrm{~m}$. This allowed us to evaluate the distance between two resonance peaks of our ring cavity (free space) as $\approx 12 \mathrm{MHz}$.

From Fig. 5, one can see that for a low incident laser power $\left(P_{1} \ll P_{\text {sat }}\right)$, the observed resonance transmittance curves are quite symmetric, as it is expected for the linear (no-saturable) fiber or for the conventional bulk FP cavities (see, e.g., Fig. 2a). In contrast, when the incident power is approaching or is higher than the saturating power of the fiber and the YDF refractive index change is significant, the resonance maxima obtain remarkable asymmetry (Fig. 5, 6a).
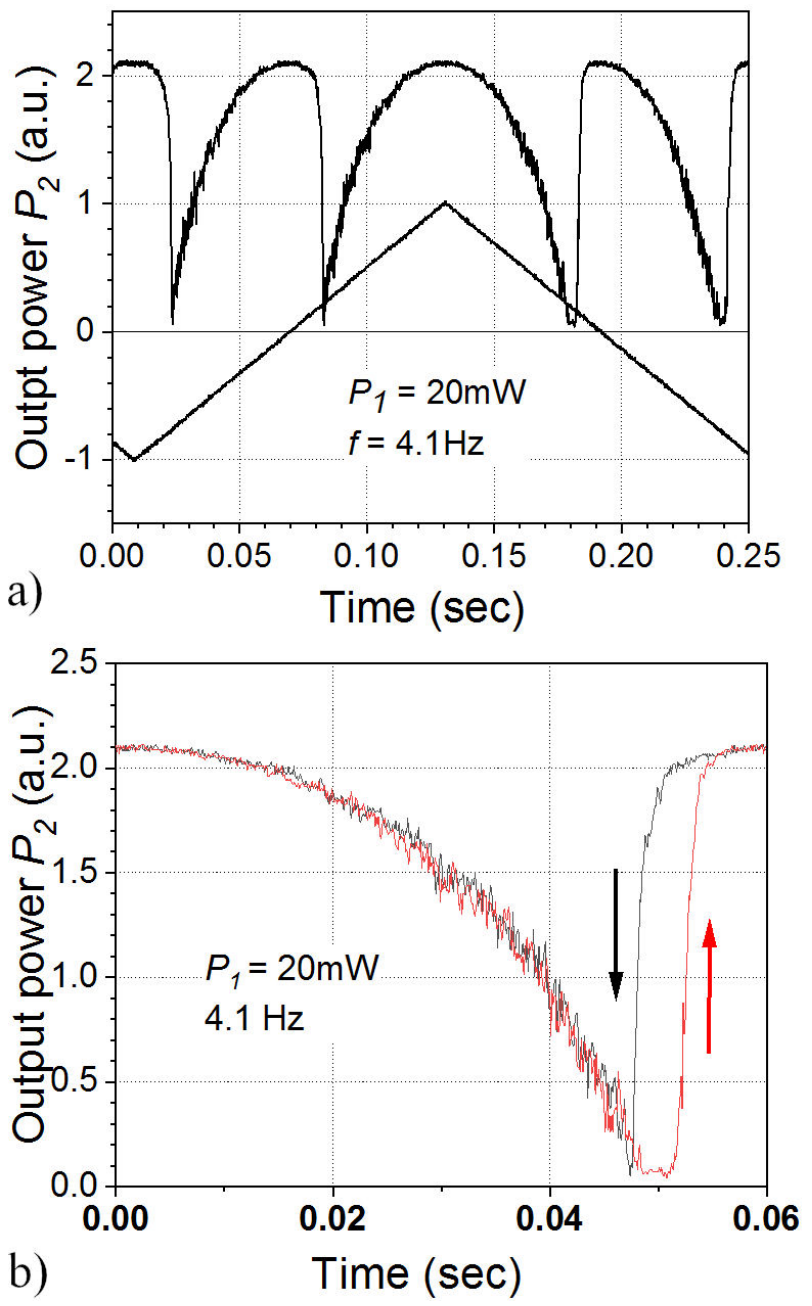

FIGURE 6. a) Transmitted power at the output of the resonance ring cavity with the saturable YDF demonstrating difference for opposite tuning scan directions (triangular profile corresponds to the modulation voltage), and b) the corresponding hysteresis loop observed for the opposite directions of the modulation voltage change.

What is important, these shape changes are different for the opposite slopes of the modulation signal -see Fig. 6a). In other words, the cavity transmittance in the resonance peak area depends on the direction one uses to cross the resonance peak. Clearly, here we observe the optical bistability effect [12] that is typical for the resonance cavities, filled with $\chi^{\prime \prime \prime}$ nonlinear optical material [13].

More clearly and directly, it is seen in Fig. 6b), where the same parts of the resonance curves from two adjacent semiperiods of the triangular modulation signal are presented as a function of the modulating voltage, but not as a function of real-time. At relatively high input light, powers one can see the hysteresis loop, where the transmittance of the nonlinear configuration depends on the direction in which we go through the resonance peak. Note that at the high cavity modulation frequency, when the YDF refractive index does not follow changes of the intracavity light power, the hysteresis 
loop disappeared, as it also does for low incident light powers.

\section{Numerical simulations}

Below we present results of the theoretical analysis and numerical simulations for the steady-state operation of the ring cavity. Note that optical bistability in nonlinear bulk FP configurations was analyzed in many earlier publications (see, e.g., [8]). The probably new element of our consideration is that in the saturable doped fiber (YDF in our case), both the refractive index and the optical absorption are changed synchronously under changing the intra-cavity light power $P_{4}$.

The basic equations utilized in our consideration are the following:

$$
\begin{aligned}
E_{2} & =E-1 \sqrt{\kappa+}+i E_{4} \sqrt{1-\kappa}\left(-\frac{\alpha L_{0}}{2}\right) \\
& \times \exp \left[i k\left(n L+\Delta n L_{0}\right)\right] \\
E_{4} & =E_{1} \sqrt{1-i \kappa+}+E_{4} \sqrt{\kappa} \exp \left(-\frac{\alpha L_{0}}{2}\right) \\
& \times \exp \left[i k\left(n L+\Delta n L_{0}\right)\right] .
\end{aligned}
$$

Here $L_{0}, \alpha$, and $\Delta n$ are the length, the saturable absorption, and the refractive index of the doped fiber segment, respectively, and $L$ and $n$ are the total length of the ring and the average (no-saturable) refractive index of the total fiber closed loop. For simplicity of consideration, we assume that the cavity is in resonance for complete saturation of the doped fiber (i.e., $k n L$ is the multiple of $2 \pi$ ). The ring optical length $L$ can be changed, however, externally by an introduction in the cavity an additional phase shift $\phi$ via the piezoelectric phase modulator (see Fig. 3).

Under this assumption, the amplitudes of the transmitted and that entering the cavity light waves can be written as:

$$
\begin{aligned}
& E_{2}=E_{1} \frac{\sqrt{\kappa}-\exp \left(\left[i \kappa \Delta n L_{0}-\frac{\alpha L_{0}}{2}\right]+i \varphi\right)}{1-\sqrt{\kappa} \exp \left(\left[i \kappa \Delta n L_{0}-\frac{\alpha L_{0}}{2}\right]+i \varphi\right)} \\
& E_{4}=E_{1} \frac{\sqrt{1-\kappa}}{1-\sqrt{\kappa} \exp \left(\left[i \kappa \Delta n L_{0}-\frac{\alpha L_{0}}{2}\right]+i \varphi\right)} .
\end{aligned}
$$

In the above equations, both the doped fiber absorption and the refractive index depend on the transmitted intracavity light power. Here we simplify the consideration and also assume that the fiber is saturated by the light power $P_{4}$ that enters the cavity. In this approximation of "constant saturating power", the exponential factors in Eqs. (3), (4) can be written as:

$$
\begin{gathered}
\exp \left(\left[i \kappa \Delta n L_{0}-\frac{\alpha L_{0}}{2}\right]+i \varphi\right) \\
=\exp [i a(\phi+i \beta)+i \varphi]
\end{gathered}
$$

Here $\phi$ and $2 \beta$ are the maximal values of the saturable phase and absorbance of the doped fiber and the saturation factor [13]:

$$
a=1 /\left(1+P_{4}\right)
$$

Here and below, in theoretical analysis, we use all the light powers normalized to the saturation power of the doped fiber.

The complex value $(\phi+i \beta)$ is a fixed parameter characteristic of this particular doped fiber segment at the selected operation wavelength. For example, it is approximately equal to $\approx i 0.6$ for the EDF at $1550 \mathrm{~nm}$ utilized in [5]. Following [15] and Fig. 3, it can be evaluated as $\approx 2.7+i 0.4$ in our 1.77 m-long YDF fiber at $1064 \mathrm{~nm}$.

Before we present the results of simulations for our particular experimental conditions, let us illustrate the analysis/simulation for two simplified cases: the purely absorbing $(\varphi=0)$ and the purely refractive $(\beta=0)$ saturable fiber. The complete analysis needs the self-consistent solution of Eq. (4)-(6) that is difficult to do analytically. Qualitatively this situation can be analyzed; however, using the graphical method [8]. To this end, we present in the same $3 \mathrm{~d}$ plot two dependencies of $P_{4}$ as functions of $a$ and $\phi$ (for the fixed selected values of $P_{1}, \kappa, \varphi$, and $\beta$ ):

$$
\begin{aligned}
& P_{4}=P_{1}\left|\frac{\sqrt{1-\kappa}}{1-\sqrt{\kappa} \exp [i a(\phi+i \beta)+i \varphi]}\right|^{2}, \\
& P_{4}=\frac{1-a}{a} .
\end{aligned}
$$

Here the first dependence is obtained from Eq. (4), and the second one-from definition of the saturation factor Eq. (6).

Example of such plot for $\kappa=0.5, \beta=0.6$, and $P_{1}=1$ is given in Fig. 7 below. In this case, the resonance peaks are

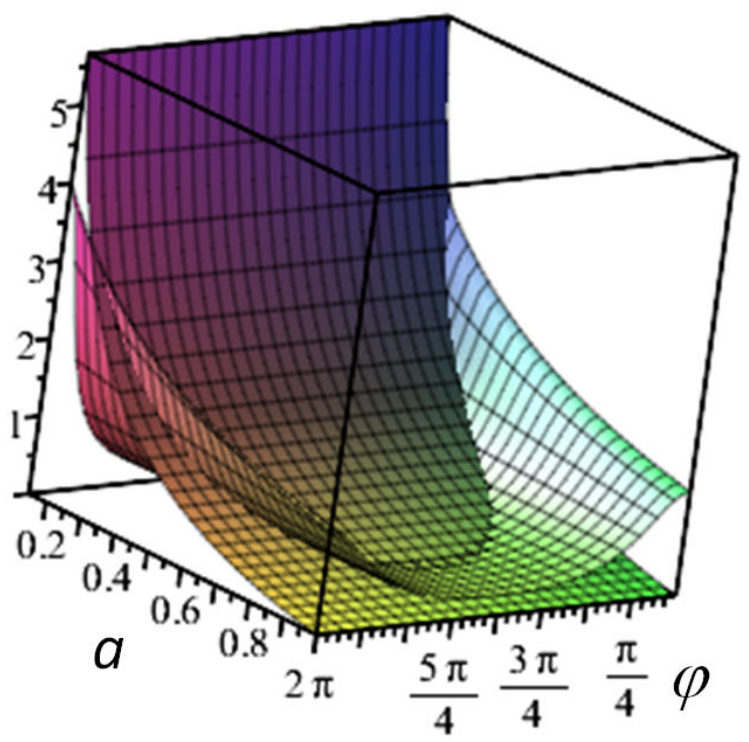

FIGURE 7. Mutual 3d plots of Eqs. (7), (8) as functions of $a$ and $\varphi$ for the fiber with saturable absorption only: $\varphi=0 \beta=0.4$, $\kappa=0.5, P_{1}=1$. 

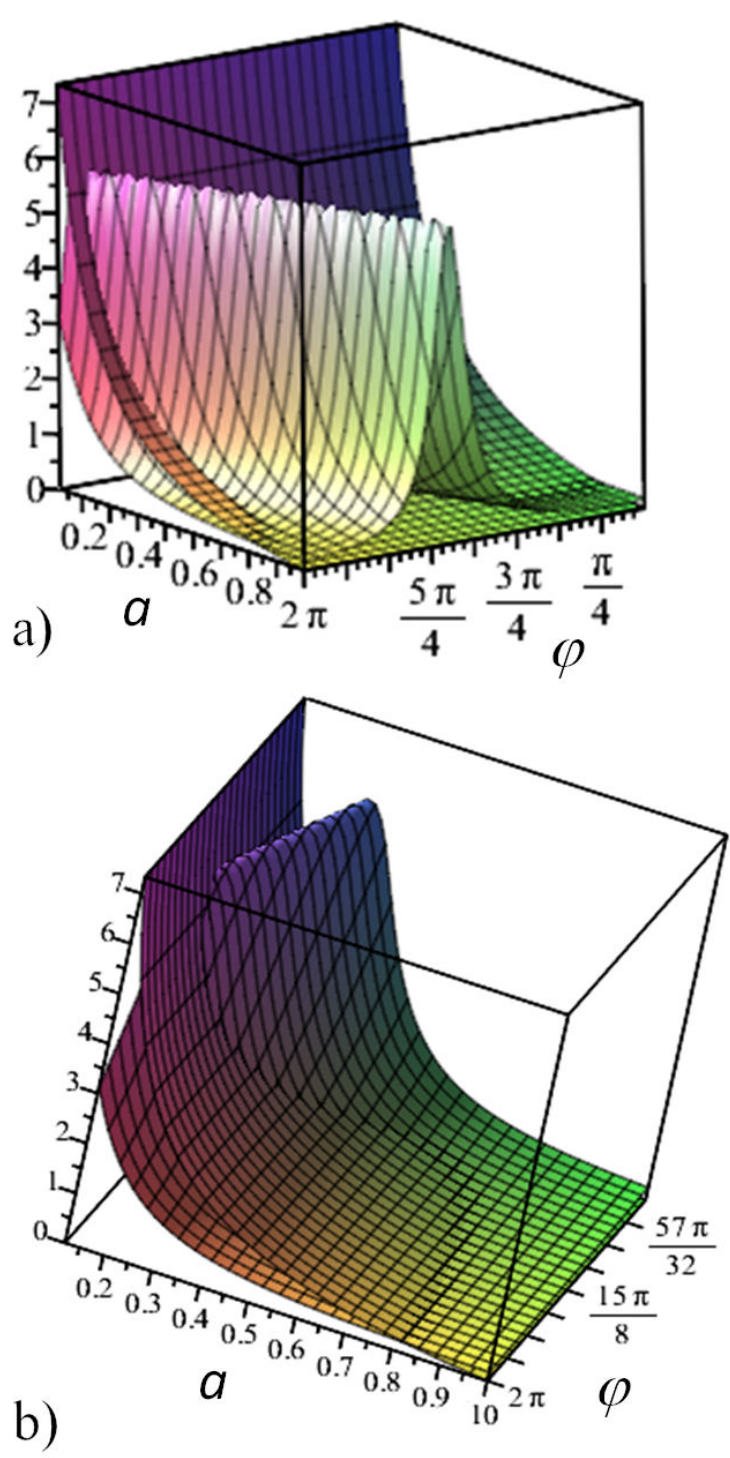

FIGURE 8. a) similar plots as in Fig. 7 for the fiber with the saturable refractive index only: $\varphi=2.7, \beta=0, \kappa=0.5, P_{1}=1$, and $b$ ) the same plot shown for the reduced range of detuning close to $\phi=2 \pi$.

always observed at $\phi=0.2 \pi$. One can see that for every fixed externally introduced cavity detuning $\phi$ (in the total range $0 \ldots 2 * P i$ ), there is one self-consistent solution for $P_{4}$ only. Clearly, this means that there is no optical bistability in this configuration.

Figure $8 \mathrm{a}$ ) below illustrates the case of a purely refractive index saturable fiber, with parameters of our YDF sample but without absorption: $\varphi=2.7, \beta=0$ evaluated for $\kappa=0.5$ and $P_{1}=1$. One can see that, in this case, the resonance peak position proves to be dependent on the initial detuning. For this reason, in a special rather narrow range of $\phi$ close to $2 \pi$-see Fig. 8b) -for one detuning, there are three solutions. It is well known that one of them (the central one) is not stable [8]; the other two correspond to two stable solutions (i.e., the bistability).
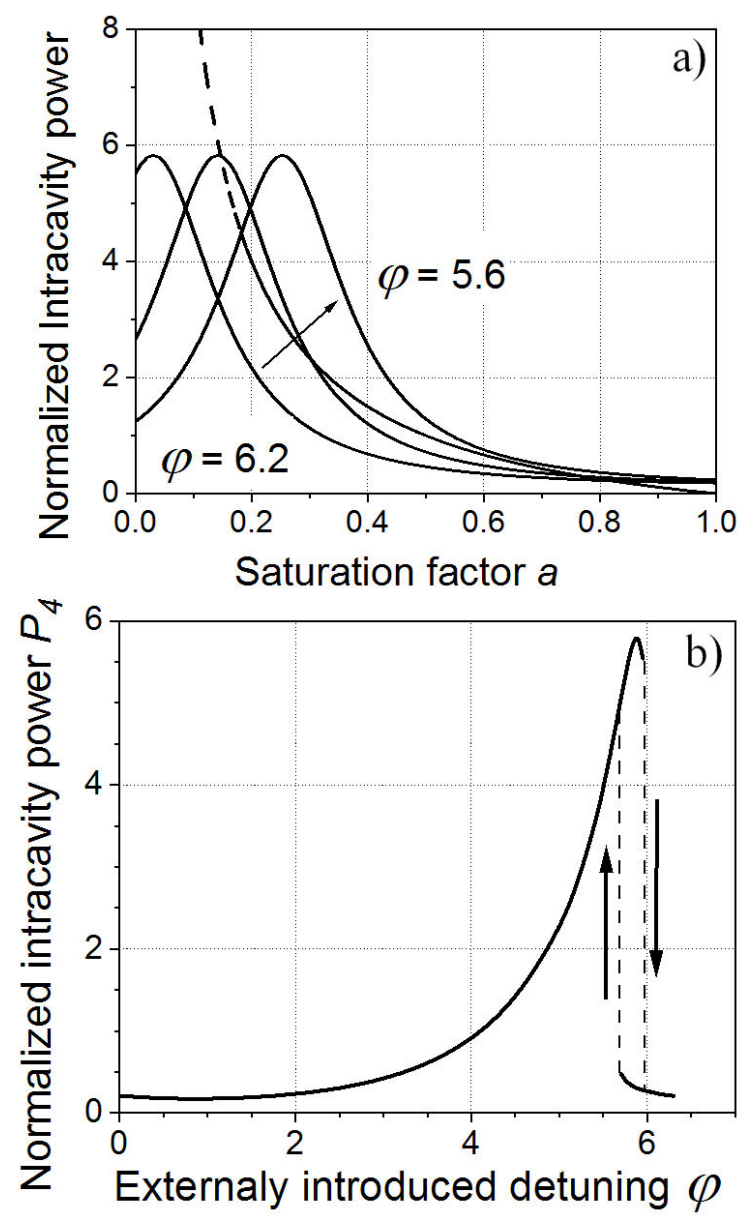

FIgURE 9. a) Cross-sections of $3 \mathrm{~d}$ plot from Fig. 8 obtained for externally introduced detunings $\phi=6.2,5.9$, and $5.6 \mathrm{rad}$ (dashed line presents the saturation factor curve Eq. 8) and b) detuning dependences of the normalized intra-cavity power obtained for the same set of parameters as in Fig. 8.

The $2 \mathrm{~d}$ cross-sections of this $3 \mathrm{~d}$ plot for several special detunings illustrating these three solutions are shown in Fig. 9a). The system occupies the state that depends on the direction from where you approach this detuning, forming, in this way, the hysteresis loop (Fig. 9b). Note that in this case, we show the hysteresis for the intra-cavity power only: because of the absence of the intra-cavity losses, the output power is always equal to the input one.

As it is illustrated in Fig. 7 itself, the saturable absorption cannot result in optical bistability in our configuration under consideration. However, because it changes the coupling of the input light with the cavity, it also changes the intra-cavity power and, as a result, can change the hysteresis loops that appear due to saturable refractive index. Corresponding $3 \mathrm{~d}$ plots calculated for the set of parameters close to that of the utilized YDF fiber $\phi+i \beta \approx 2.7+i 0.4$ and $P_{1}=1$ are shown in Fig. 10.

From these plots, we can reconstruct the detuning dependences of the output and the intra-cavity light powers that are presented in Fig. 11. As expected, the transmitted power tends to the conventional symmetric shape of the detuning 

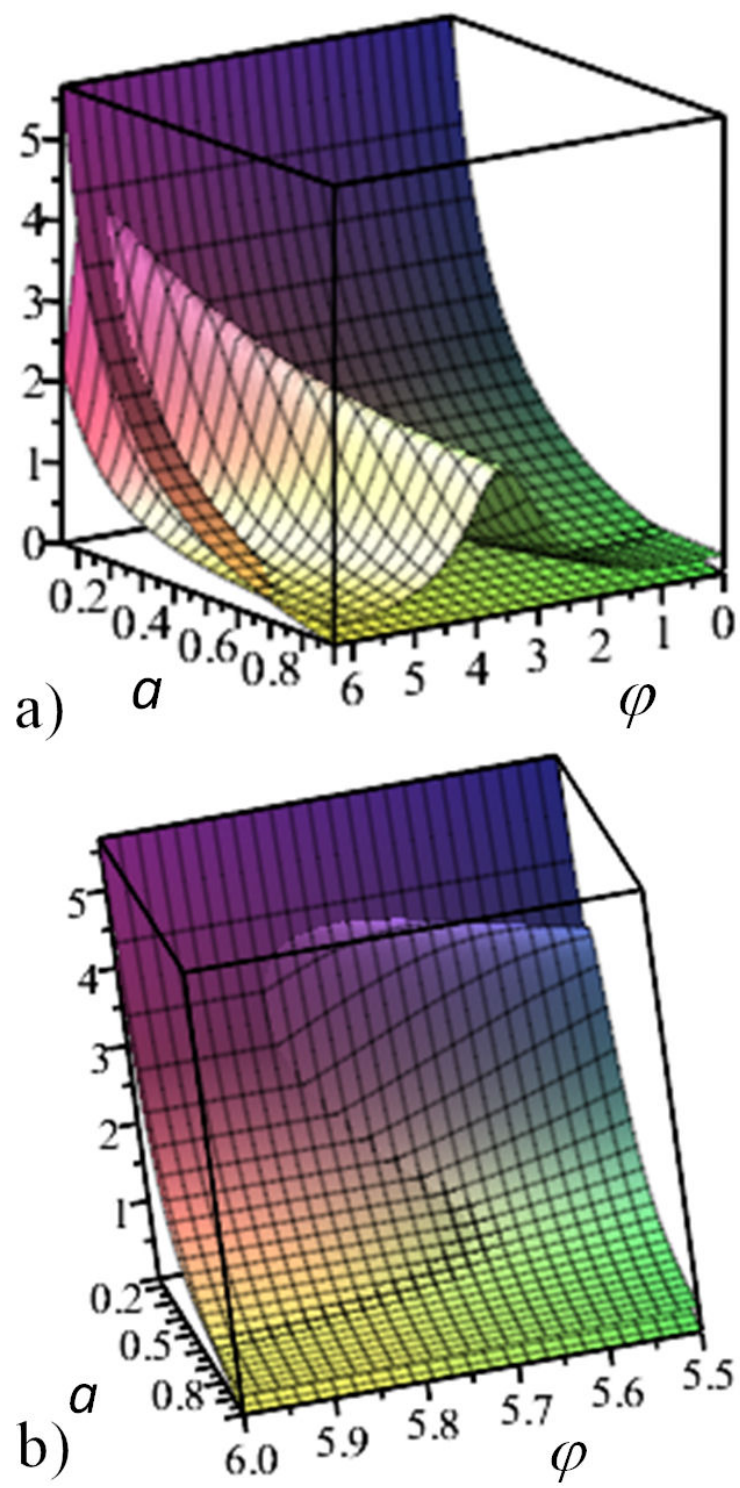

FIGURE 10. $3 \mathrm{~d}$ plots similar to those in Fig. 8 for the saturable medium with parameters close to those of the utilized YDF fiber: $\varphi=2.7, \beta=0.4, \kappa=0.5, P_{1}=1$.

curve for the incident light power significantly lower than the saturation power. The hysteresis loop is most pronounced for the incident light power close to the saturation power of the doped fiber.

One can see that, qualitatively, the results of our numerical simulation correspond to the presented experimental results (Fig. 6, 7). In particular, one can observe significant asymmetry of the tuning transmittance curves for the incident powers approaching and higher than the saturation power. The experimentally observed hysteresis loop width (Fig. 7) proves, however, significantly larger than the theoretical evaluation of $\approx 0.2$ rad that can be obtained, in particular, from Fig. 11b). This disagreement can partially be attributed to a relatively high speed of scanning through the resonance cavity. Additionally, it is necessary to introduce into the simulations possible light attenuation introduced by the other intra-
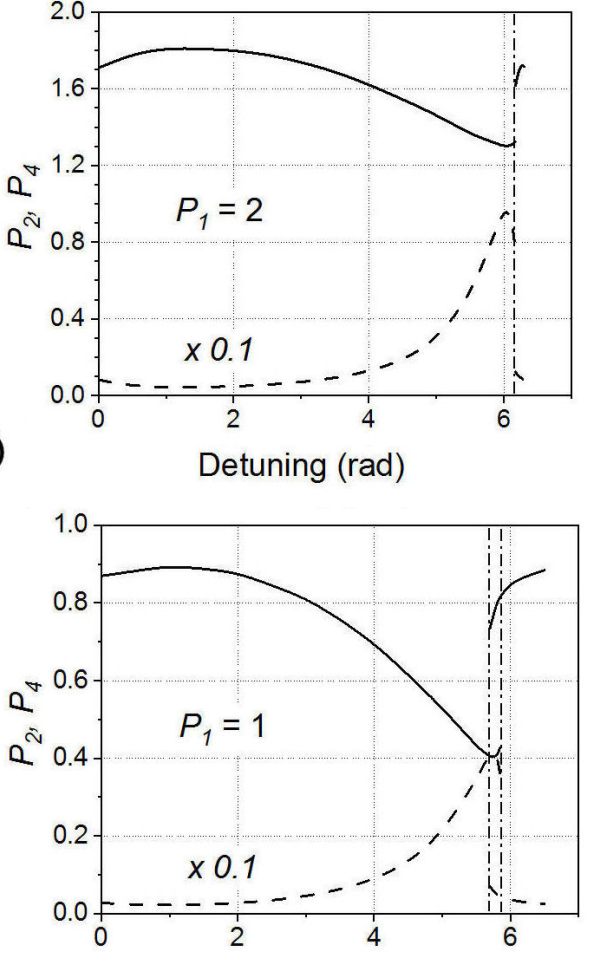

b)

Detuning (rad)
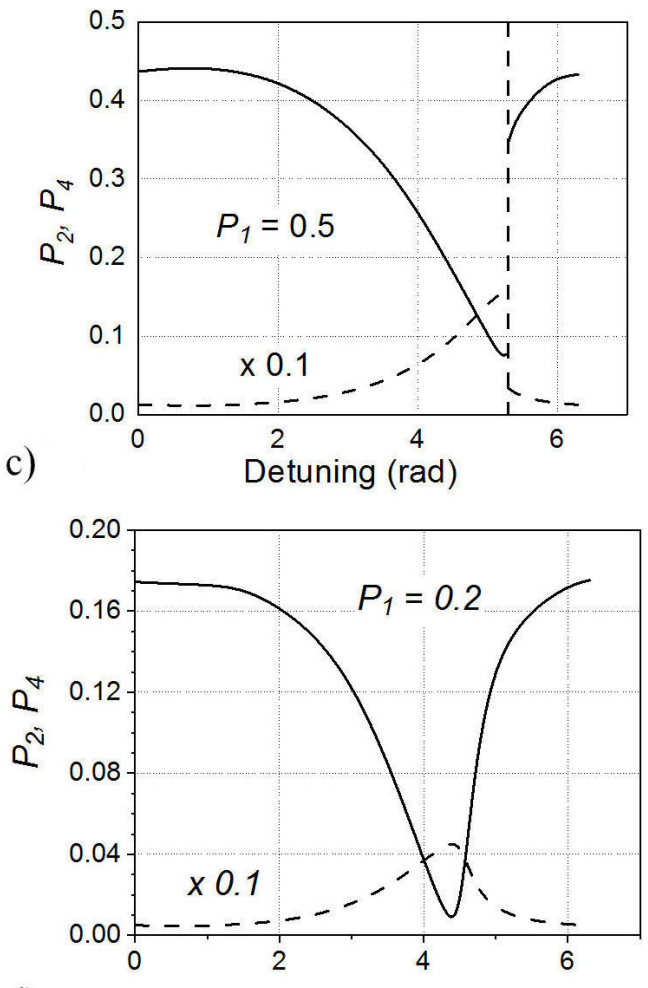

d)

Detuning (rad)

FIGURE 11. Detuning dependences of the normalized output (solid lines) and the intra-cavity (dashed lines) power, calculated for the saturable medium with parameters of the utilized YDF fiber: $\varphi=2.7, \beta=0.4, \kappa=0.5$ and for the normalized incident power a) $P_{1}=2$, b) 1 , c) 0.5 , and d) 0.2 . 
cavity elements. Surely, it is also necessary to evaluate more precisely the doped fiber nonlinearity.

During an initial period of investigations of the optical bi- and multi-stability, these effects were considered promising for the development of the optical logic and memory devices [8]. However, the high potential of these effects for improving the performance of the optical interferometric measuring configurations was also estimated. Now such applications of similar arrangements are considered highly promising; especially, when utilization and generation of the nonclassical light are involved [16,17].

Simply this important property can be illustrated using the response curves similar to those presented in Figs. 11c) d). One can see that at the right side of the resonance transmittance curve, the slope angle can theoretically be very high, as compared with that at the other, left side, or in case, of the resonance curve of the linear FP cavity (Fig. 2a). This means that a small phase modulation introduced in a non-linear cavity, tuned to this special point, can result in an unlimited response in the transmitted light power.

Note that the necessary asymmetric shape of the resonance transmittance curve with highly steep one side was indeed, observed in our experiments -see Fig. 5. The experiments on intra-cavity sinusoidal phase modulation under such resonance conditions are in progress in our group now. Here it is necessary to take into account that the theoretical response curves (Fig. 11) were obtained for the quasi-steadystate conditions, i.e., when the phase modulation signals are significantly slower than the characteristic time of the non- liner (saturation) response. In the case of the utilized saturable YDF, this implies an operation frequency range below some fraction of $1 \mathrm{kHz}$.

\section{Conclusions}

Summarizing, we have presented original experimental results on the investigation of the ring fiber-optic resonance cavity with the saturable ytterbium-doped fiber. In particular, we have demonstrated the optical bistability and the optical hysteresis for the incident $\mathrm{cw}$ power close to the saturation power of the YDF at $1064 \mathrm{~nm}(\sim 10 \mathrm{~mW})$. We associate the observed effects with a high photo-induced response in the saturable refractive index of the doped fiber. The presented preliminary results are in a qualitative agreement with the theoretical analysis and with numerical simulation. We hope that the presented experimental configurations can be promising for applications in high-sensitivity fiber configurations operating at $1064 \mathrm{~nm}$.

One of the co-authors of this text, namely SS, wants to express his deep regret about the unexpected death of his very close personal friend, highly respected person, excellent researcher-physicist, and the real Teacher for many of his students -Dr. Evgeny Kuzin.

\section{Funding}

Consejo Nacional de Ciencia y Tecnología (CONACYT) (CB-2014-01-239941).
1. L. F. Stokes, S. Chodorow, and H. J. Shaw, All-single-mode fiber resonators, Opt. Lett. 7 (1982) 288-290. https:// doi.org/10.1364/OL.7.000288

2. J. M. Choi, R. K. Lee, and A. Yariv,Control of critical coupling in a ring resonator-fiber configuration: application to wavelength-selective switching, modulation, amplification, and oscillation, Opt. Lett. 26 (2001) 1236-1238. https: / / doi. org/10.1364/OL.26.001236

3. N. Langford, Narrow-Linewidth Fiber Lasers in Rare-EarthDoped Fiber Lasers and Amplifiers, ed by M. J. F. Digonnet (Marcel Dekker, NY, 2001) pp. 243-340.

4. J. E. Heebner, V. Wong, A. Schweinsberg, R. W. Boyd, and D. J. Jackson, Optical transmission characteristics of fiber ring resonator, IEEE J. Quantum Electron. 40 (2004) 726-730. $10.1109 / \mathrm{JQE} .2004 .828232$

5. A. Shlyagina and S. Stepanov, Optically controlled lightpulse propagation in closed-loop configuration with saturable erbium-doped fiber JOSA B 31 (2014) 2414-2418. https: //doi.org/10.1364/JOSAB.31.002414

6. M. Tomita, T. Ueta, and P. Sultana, Slow optical pulse propagation in an amplifying ring resonator, JOSA B 28 (2011) 1627-1630. https://doi.org/10.1364/JOSAB.28. 001627
7. Y. O. Barmenkov, A. V. Kir'yanov, and M. V. Andrés, Resonant and thermal changes of refractive index in a heavily doped erbium fiber pumped at wavelength $980 \mathrm{~nm}$, Appl. Phys. Lett. 85 (2004) 2466-2468. https://doi.org/10.1063/1. 1787151

8. H. M. Gibbs, Optical bistability: Controlling light with light (Academic Press, Orlando, 1985).

9. T. N. C. Venkatesan and S. L. McCall, Optical bistability and differential gain between 85 and $296 \mathrm{~K}$ in a Fabry-Perot containing ruby Appl. Phys. Lett. 30 (1977) 282-284. https: //doi.org/10.1063/1.89368

10. A. A. Fotiadi, O. L. Antipov, and P. Mégret, Dynamics of pump-induced refractive index changes in single-mode $\mathrm{Yb}$ doped optical fibers, Opt. Express 16 (2008) 12658-12663. https://doi.org/10.1364/OE.16.012658

11. E. Aguilar, S. Stepanov, and E. Hernandez, High-resolution adaptive interferometer with dynamic population grating recorded at $1064 \mathrm{~nm}$ in Ytterbium-doped fiber Appl.Opt. 59 (2020) 6131-6137. https://doi.org/10.1364/AO. 394478

12. R. Paschotta, J. Nilsson, A. C. Tropper, and D. C. Hanna, Ytterbium doped fiber amplifiers, IEEE J. Quantum Electron. 33 (1997) 1049-1056. DOI : $10.1109 / 3.594865$ 
13. A. E. Siegman, Lasers (University Science Books, Sausalito, 1986).

14. S. Stepanov, A. Fotiadi, and P. Megret, Effective recording of dynamic phase gratings in Yb-doped fibers with saturable absorption at $1064 \mathrm{~nm}$ Opt. Express 15 (2007) 8832-8837. https://doi.org/10.1364/OE.15.008832

15. S. Stepanov and M. Plata Sánchez, Phase population gratings recorded in ytterbium doped fiber at 1064nm SPIE Proc. 8011 (2011) 1 .
16. H. Rehbein, J. Harms, R. Schnabel, and K. Danzmann, Optical transfer function of Kerr nonlinear cavities and interferometers Phys. Rev. Lett. 95 (2005) 103001. https : / / doi.org/10. 1103/PhysRevLett.95.193001

17. A. Khalaidovski, A. Thuring, H. Rehbein, N. Lastzka, B. Willke, K. Danzmann, and R. Schnabel, Strong reduction of laser power noise by means of a Kerr nonlinear cavity. Phys. Rev. A 80 (2009) 053801. 